\title{
EXTENSION OF THE HILBERT TRANSFORM
}

\author{
Holger Boche and Ullrich J. Mönich \\ Technische Universität München \\ Lehrstuhl für Theoretische Informationstechnik \\ 80290 München, Germany
}

\begin{abstract}
The Hilbert transform is an important operator in signal processing, e.g., the definition of the "analytical signal" uses the Hilbert transform. In this paper we analyze the Hilbert transform for bounded bandlimited signals in $\mathcal{B}_{\pi}^{\infty}$. Although the common integral representation of the Hilbert transform may diverge for certain signals in $\mathcal{B}_{\pi}^{\infty}$, it is possible to define the Hilbert transform meaningfully for bounded signals. We employ a definition that is based on the $\mathcal{H}^{1}-\mathrm{BMO}(\mathbb{R})$ duality. The problem of this abstract definition is that there exists no constructive procedure to calculate the Hilbert transform. However, for the subspace of bounded bandlimited signals, we can give an explicit formula for the calculation of the Hilbert transform. Further, we show that the Hilbert transform of a bounded bandlimited signal is still bandlimited but not necessarily bounded.
\end{abstract}

Index Terms - Hilbert transform, bounded bandlimited signal, bounded mean oscillation, Hardy space

\section{INTRODUCTION}

In signal processing and communication theory the Hilbert transform is an important operator with many applications $[1,2,3,4,5]$. For example, the calculation of the analytical signal, which was introduced by Gabor in his "Theory of Communication" [1], requires the Hilbert transform. In an analytical signal $\psi(t)=u(t)+i v(t)$ the imaginary part $v$ is the Hilbert transform of the real part $u$. Based on the analytical signal it is possible to define the instantaneous amplitude and frequency of a signal [3,4]. Although there are other possibilities to define the instantaneous amplitude and frequency [4], it was shown in [4] that the only definition that satisfies certain physical requirements is the definition based on the Hilbert transform and the analytical signal.

Classically, the Hilbert transform of a signal $f$ is defined as the principal value integral

$$
\begin{aligned}
(H f)(t) & =\frac{1}{\pi} \text { V.P. } \int_{-\infty}^{\infty} \frac{f(\tau)}{t-\tau} \mathrm{d} \tau \\
& =\frac{1}{\pi} \lim _{\epsilon \rightarrow 0}\left(\int_{t-\frac{1}{\epsilon}}^{t-\epsilon} \frac{f(\tau)}{t-\tau} \mathrm{d} \tau+\int_{t+\epsilon}^{t+\frac{1}{\epsilon}} \frac{f(\tau)}{t-\tau} \mathrm{d} \tau\right) .
\end{aligned}
$$

Of course, the above definition of the Hilbert transform only makes sense if the integral converges. Numerous authors have studied the convergence behavior of the integral, and it is known that there exist bounded signals for which the integral in (1) diverges everywhere. Even if the signal is additionally bandlimited, the integral (1)

This work was supported by start-up funds of the Technische Universität München and the German Research Foundation (DFG) under grant BO 1734/21-1. may diverge. However, for the subclass of bounded bandpass signals, the Hilbert transform exists and is bounded. If $f$ is a bandpass signals, the distributional Fourier transform of which vanishes outside $[-\pi,-\epsilon \pi] \cup[\epsilon \pi, \pi], 0<\epsilon<1$, then $f$ has a bounded Hilbert transform satisfying $\|H f\|_{\infty} \leq\left(A+\frac{2}{\pi} \log \left(\frac{1}{\epsilon}\right)\right)\|f\|_{\infty}$, where $A<4 / \pi$ is a constant [6,7]. Probably, observations of this kind led to the conclusion "that an arbitrary bounded bandlimited function does not have a Hilbert transform..." [7].

Since then, several extension of the Hilbert transformation have been proposed and analyzed [8, 9, pp. 44]. For example, the Hilbert transform for general groups [10] or extensions to bounded functions [9]. In [3] a set of conditions was given which is sufficient for the convergence of (1) to a continuous and bounded signal. However, this set of conditions is not necessary for the convergence of (1) to a continuous and bounded signal, and it is difficult to characterize the class of bounded signals that have a bounded Hilbert transform. As for finding an extension of the Hilbert for bounded bandlimited signals, essentially no progress has been made since Logan published his paper [7] in 1978.

In this paper we use an extension of the Hilbert transform which is based on the $\mathcal{H}^{1}$-BMO $(\mathbb{R})$ duality. This extension, which will be introduced in Section 5, is valid for general bounded signals, however, provides no constructive procedure for the calculation of the Hilbert transform. In Section 6 we show that, for the subspace $\mathcal{B}_{\pi}^{\infty}$ of bounded bandlimited signals, there exists an explicit formula for the calculation of the Hilbert transform. An integral part of this formula is an operator that consists of the concatenation of the Hilbert transform and the differential operator. The properties of this compound operator are discussed in Section 4.

\section{NOTATION}

Let $\hat{f}$ denote the Fourier transform of a function $f . L^{p}(\mathbb{R}), 1 \leq$ $p<\infty$, is the space of all $p$ th-power Lebesgue integrable functions on $\mathbb{R}$, with the usual norm $\|\cdot\|_{p}$, and $L^{\infty}(\mathbb{R})$ is the space of all functions for which the essential supremum norm $\|\cdot\|_{\infty}$ is finite. For $0<\sigma<\infty$ let $\mathcal{B}_{\sigma}$ be the set of all entire functions $f$ with the property that for all $\epsilon>0$ there exists a constant $C(\epsilon)$ with $|f(z)| \leq$ $C(\epsilon) \exp ((\sigma+\epsilon)|z|)$ for all $z \in \mathbb{C}$. The Bernstein space $\mathcal{B}_{\sigma}^{p}, 1 \leq$ $p \leq \infty$, consists of all functions in $\mathcal{B}_{\sigma}$, whose restriction to the real line is in $L^{p}(\mathbb{R})$. The norm for $\mathcal{B}_{\sigma}^{p}$ is given by the $L^{p}$-norm on the real line, i.e., $\|\cdot\|_{\mathcal{B}_{\sigma}^{p}}=\|\cdot\|_{p}$. A signal in $\mathcal{B}_{\sigma}^{p}$ is called bandlimited to $\sigma$, and $\mathcal{B}_{\sigma}^{\infty}$ is the space of bandlimited signals that are bounded on the real axis. We call a signal in $\mathcal{B}_{\pi}^{\infty}$ bounded bandlimited signal. Moreover, $\mathcal{B}_{\sigma, 0}^{\infty}$ denotes the set of all signals in $\mathcal{B}_{\sigma}^{\infty}$ that vanish on the real axis at infinity. For $0<\sigma<\infty$ and $1 \leq p \leq \infty$ we denote by $\mathcal{P} \mathcal{W}_{\sigma}^{p}$ the Paley-Wiener space of signals $f$ with a representation $f(z)=1 /(2 \pi) \int_{-\sigma}^{\sigma} g(\omega) \mathrm{e}^{i z \omega} \mathrm{d} \omega, z \in \mathbb{C}$, for some $g \in L^{p}[-\sigma, \sigma]$. 
If $f \in \mathcal{P} \mathcal{W}_{\sigma}^{p}$ then $g(\omega)=\hat{f}(\omega)$. The norm for $\mathcal{P} \mathcal{W}_{\sigma}^{p}, 1 \leq p<\infty$, is given by $\|f\|_{\mathcal{P} \mathcal{W}_{\sigma}^{p}}=\left(1 /(2 \pi) \int_{-\sigma}^{\sigma}|\hat{f}(\omega)|^{p} \mathrm{~d} \omega\right)^{1 / p}$. For $p=2$ we obtain the Paley-Wiener space $\mathcal{P} \mathcal{W}_{\sigma}^{2}=\mathcal{B}_{\pi}^{2}$, which is nothing else than the space of bandlimited signals with finite energy.

We briefly review some facts about stable LTI systems. A linear system $T: \mathcal{A} \rightarrow \mathcal{B}$, mapping signals from the Banach space $\mathcal{A}$ to the Banach space $\mathcal{B}$, is called stable if the operator $T$ is bounded, i.e., if $\|T\|=\sup _{\|f\|_{\mathcal{A}} \leq 1}\|T f\|_{\mathcal{B}}<\infty$. Furthermore, it is called time invariant if $(T f(\cdot-a))(t)=(T f)(t-a)$ for all $f \in \mathcal{A}$ and $t, a \in \mathbb{R}$. The Hilbert transform $H: \mathcal{P W}_{\pi}^{2} \rightarrow \mathcal{P W}_{\pi}^{2}$ is an example of a stable LTI system, which has many applications, e.g., in signal processing and communication theory [11]. One possibility to define the Hilbert transform $H$ for signals $f \in \mathcal{P} \mathcal{W}_{\pi}^{2}$ is given by $(H f)(t):=\frac{1}{2 \pi} \int_{-\pi}^{\pi}(-i \operatorname{sgn}(\omega)) \hat{f}(\omega) \mathrm{e}^{i \omega t} \mathrm{~d} \omega$, where sgn denotes the signum function. Another example for an stable LTI system is the differential operator $D: \mathcal{P} \mathcal{W}_{\pi}^{2} \rightarrow \mathcal{P} \mathcal{W}_{\pi}^{2}$, which is defined by $(D f)(t):=\frac{1}{2 \pi} \int_{-\pi}^{\pi} i \omega \hat{f}(\omega) \mathrm{e}^{i \omega t} \mathrm{~d} \omega$.

\section{MOTIVATION}

For all signals in the $L^{p}(\mathbb{R})$ spaces with $1<p<\infty$, the integral in (1) converges for almost all $t \in \mathbb{R}$, and the Hilbert transform $H: L^{p}(\mathbb{R}) \rightarrow L^{p}(\mathbb{R})$ is a bounded linear operator [12, pp. 255]. In this paper we are interested in the behavior of the Hilbert transform for bandlimited signals that are bounded on the real axis, i.e., signals in $\mathcal{B}_{\pi}^{\infty}$.

It is well-known that the classical Hilbert transform $H$ as defined in (1) is no bounded operator on $\mathcal{B}_{\pi}^{\infty}$ [6]. The following example illustrates this behavior. Consider the family of $\mathcal{P} \mathcal{W}_{\pi}^{2}$-signals $\left\{f_{\epsilon}\right.$ : $0<\epsilon<\pi\}$ given by

$$
f_{\epsilon}(t)=-\frac{2}{\pi} \int_{\epsilon}^{\pi} \frac{\sin (\omega t)}{\omega} \mathrm{d} \omega, \quad t \in \mathbb{R} .
$$

We have $\left\|f_{\epsilon}\right\|_{\infty} \leq C_{1}$ with a constant $C_{1}$ that is independent of $\epsilon$, and the limit signal

$$
f_{\mathrm{L}}(t)=\lim _{\epsilon \rightarrow 0} f_{\epsilon}(t)=-\frac{2}{\pi} \int_{0}^{\pi} \frac{\sin (\omega t)}{\omega} \mathrm{d} \omega
$$

is a signal in $\mathcal{B}_{\pi}^{\infty}$. However, we have that $\lim _{\epsilon \rightarrow 0}\left(H f_{\epsilon}\right)(t)=\infty$ for all $t \in \mathbb{R}$. In particular, we have $\left(H f_{\epsilon}\right)(0) \geq \frac{2}{\pi} \log \left(\frac{\pi}{\epsilon}\right)$. Thus, the Hilbert transform operator $H$ as defined in (1) is an unbounded operator on $\mathcal{B}_{\pi}^{\infty}$. In fact, there are signals in $\mathcal{B}_{\pi}^{\infty}$ for which the principal value integral definition of the Hilbert integral is meaningless, because (1) diverges everywhere [13].

Note that this result does not imply that the Hilbert transform cannot be defined for the space $\mathcal{B}_{\pi}^{\infty}$. There may be other definitions, apart from the principal value integral definition (1), that are meaningful. In Section 5 we will present such a meaningful definition of the Hilbert transformation for the space $\mathcal{B}_{\pi}^{\infty}$.

\section{THE OPERATOR $Q$}

In this section we consider the compound LTI system $Q=D H$, which consists of the concatenation of the Hilbert transform $H$ and the differential operator $D$. First, we consider $Q$ as an operator acting on $\mathcal{P} \mathcal{W}_{\pi}^{2}$. Since both operators $H: \mathcal{P} \mathcal{W}_{\pi}^{2} \rightarrow \mathcal{P} \mathcal{W}_{\pi}^{2}$ and $D: \mathcal{P} \mathcal{W}_{\pi}^{2} \rightarrow \mathcal{P} \mathcal{W}_{\pi}^{2}$ are stable LTI systems, $Q: \mathcal{P} \mathcal{W}_{\pi}^{2} \rightarrow \mathcal{P} \mathcal{W}_{\pi}^{2}$, as the concatenation of two stable LTI systems, is a stable LTI system. The system $Q: \mathcal{P} \mathcal{W}_{\pi}^{2} \rightarrow \mathcal{P} \mathcal{W}_{\pi}^{2}$ has the frequency domain representation

$$
(Q f)(t)=(D H f)(t)=\frac{1}{2 \pi} \int_{-\pi}^{\pi} \hat{h}_{Q}(\omega) \hat{f}(\omega) \mathrm{e}^{i \omega t} \mathrm{~d} \omega,
$$

where

$$
\hat{h}_{Q}(\omega)= \begin{cases}|\omega|, & |\omega| \leq \pi \\ 0, & |\omega|>\pi\end{cases}
$$

Two other possible representations for the system $Q$ are given by the time-domain convolution integral

$$
(Q f)(t)=\int_{-\infty}^{\infty} f(\tau) h_{Q}(t-\tau) \mathrm{d} \tau
$$

and the time-domain sampling representation

$$
(Q f)(t)=\sum_{k=-\infty}^{\infty} f(k) h_{Q}(t-k),
$$

where $h_{Q}$ is given by

$$
\begin{aligned}
h_{Q}(t) & =(Q \operatorname{sinc})(t)=\frac{1}{2 \pi} \int_{-\pi}^{\pi} \hat{h}_{Q}(\omega) \mathrm{e}^{i \omega t} \mathrm{~d} \omega \\
& =\frac{\pi t \sin (\pi t)+\cos (\pi t)-1}{\pi t^{2}} .
\end{aligned}
$$

Compared to the convolution integral representation (5), the sampling representation (6) has the advantage that only the samples $\{f(k)\}_{k \in \mathbb{Z}}$ of the signal $f$ are needed in order to calculate $(Q f)(t)$, $t \in \mathbb{R}$. In contrast, in the representation (5) all signal values $f(t)$, $t \in \mathbb{R}$, are necessary.

\subsection{Mixed Signal Representation}

Next, we show that in addition to the convolution integral representation (5) and the convolution sum representation (6), the systems $Q: \mathcal{P} \mathcal{W}_{\pi}^{2} \rightarrow \mathcal{P} \mathcal{W}_{\pi}^{2}$ has the mixed signal representation

$$
(Q f)(t)=\sum_{k=-\infty}^{\infty} a_{-k} f(t-k),
$$

where $a_{-k}, k \in \mathbb{Z}$, are certain coefficients. We call this representation mixed signal representation, because for a fixed $t \in \mathbb{R}$ we need the signal values on the discrete grid $\{t-k\}_{k \in \mathbb{Z}}$ in order to calculate $(Q f)(t)$. However, for different $t \in \mathbb{R}$ we need other signal values in general. As $t$ ranges over $[0,1]$ we need all the signal values $f(\tau)$, $\tau \in \mathbb{R}$. The mixed signal representation (8) will be important for the results in Section 6, where we extend the Hilbert transform to $\mathcal{B}_{\pi}^{\infty}$.

In order to see the validity of the mixed signal representation (8), we consider the Fourier series of the $2 \pi$-periodic extension of $\hat{h}_{Q}$, i.e.,

$$
\sum_{k=-\infty}^{\infty} a_{k} \mathrm{e}^{i \omega k}
$$

where the coefficients $a_{k}, k \in \mathbb{Z}$, are given by

$$
a_{k}=\frac{1}{2 \pi} \int_{-\pi}^{\pi}|\omega| \mathrm{e}^{i \omega k} \mathrm{~d} \omega= \begin{cases}\frac{\pi}{2}, & k=0, \\ \frac{(-1)^{k}-1}{\pi k^{2}}, & k \neq 0 .\end{cases}
$$

Since,

$$
\begin{aligned}
\sum_{k=-\infty}^{\infty}\left|a_{k}\right| & =\frac{\pi}{2}+2 \sum_{k=1}^{\infty} \frac{\left|(-1)^{k}-1\right|}{\pi k^{2}} \\
& =\frac{\pi}{2}+\frac{4}{\pi} \sum_{k=1}^{\infty} \frac{1}{(2 k-1)^{2}}=\pi
\end{aligned}
$$


we see that the Fourier series (9) is absolutely and uniformly convergent. Hence, starting with the frequency domain representation (4) of $Q f$, we obtain

$$
\begin{aligned}
(Q f)(t) & =\frac{1}{2 \pi} \int_{-\pi}^{\pi} \hat{f}(\omega) \hat{h}_{Q}(\omega) \mathrm{e}^{i \omega t} \mathrm{~d} \omega \\
& =\frac{1}{2 \pi} \int_{-\pi}^{\pi} \hat{f}(\omega)\left(\sum_{k=-\infty}^{\infty} a_{k} \mathrm{e}^{i \omega k}\right) \mathrm{e}^{i \omega t} \mathrm{~d} \omega \\
& =\sum_{k=-\infty}^{\infty} a_{-k} \frac{1}{2 \pi} \int_{-\pi}^{\pi} \hat{f}(\omega) \mathrm{e}^{i \omega(t-k)} \mathrm{d} \omega \\
& =\sum_{k=-\infty}^{\infty} a_{-k} f(t-k)
\end{aligned}
$$

for all $t \in \mathbb{R}$. The exchange of the integral and the sum in (12) was justified because the series (9) is uniformly convergent on $[-\pi, \pi]$.

\subsection{Extension of $Q$ to $\mathcal{B}_{\pi}^{\infty}$}

So far we have considered the LTI system $Q$ only acting on signals in $\mathcal{P} \mathcal{W}_{\pi}^{2}$. An interesting question is whether $Q$ can be meaningfully extended to a bounded operator $Q^{\mathrm{E}}$ acting on the larger space $\mathcal{B}_{\pi}^{\infty}$ of bandlimited signals that are bounded on the real axis. For the operator $Q: \mathcal{P} \mathcal{W}_{\pi}^{2} \rightarrow \mathcal{P} \mathcal{W}_{\pi}^{2}$ we had the four different representations (4)-(6) and (8). However, the frequency domain representations which involves the Fourier transform of the signal makes no sense for signals in $\mathcal{B}_{\pi}^{\infty}$. The next theorem shows that the mixed signal representation (8) is still meaningful for signals in $\mathcal{B}_{\pi}^{\infty}$, because

$$
Q^{\mathrm{E}} f=\sum_{k=-\infty}^{\infty} a_{-k} f(t-k),
$$

is also a valid representation of the operator $Q^{\mathrm{E}}: \mathcal{B}_{\pi}^{\infty} \rightarrow \mathcal{B}_{\pi}^{\infty}$.

Theorem 1. There exists a bounded linear operator $Q^{E}: \mathcal{B}_{\pi}^{\infty} \rightarrow$ $\mathcal{B}_{\pi}^{\infty}$ with norm $\left\|Q^{E}\right\|=\pi$ that coincides with $Q$ on $\mathcal{P} \mathcal{W}_{\pi}^{2}$, i.e., that satisfies $Q^{E} f=Q f$ for all $f \in \mathcal{P} \mathcal{W}_{\pi}^{2}$.

Proof. We will show that the mixed signal representation (13), where the coefficients $a_{k}$ are defined as in (10), defines a bounded linear operator that maps $\mathcal{B}_{\pi}^{\infty}$ into $\mathcal{B}_{\pi}^{\infty}$, and thus gives us the desired extension. The linearity of $Q^{\mathrm{E}}$ is obvious. It remains to show that the norm of $Q^{\mathrm{E}}$ satisfies $\left\|Q^{\mathrm{E}}\right\|=\pi$. Since $\left|\left(Q^{\mathrm{E}} f\right)(t)\right| \leq$ $\|f\|_{\infty} \sum_{k=-\infty}^{\infty}\left|a_{k}\right|$ for all $t \in \mathbb{R}$, it follows from (11) that $\left\|Q^{\mathrm{E}} f\right\|_{\infty} \leq \pi\|f\|_{\infty}$. Moreover, for $t \in \mathbb{R}$, we have

$$
\begin{aligned}
\left(Q^{\mathrm{E}} \mathrm{e}^{i \pi \cdot}\right)(t) & =\sum_{k=-\infty}^{\infty} a_{-k} \mathrm{e}^{i \pi(t-k)}=\sum_{k=-\infty}^{\infty} a_{-k} \mathrm{e}^{-i \pi k} \mathrm{e}^{i \pi t} \\
& =\hat{h}_{Q}(\pi) \mathrm{e}^{i \pi t}=\pi \mathrm{e}^{i \pi t},
\end{aligned}
$$

which shows that $\left\|Q^{\mathrm{E}}\right\| \geq\left\|Q^{\mathrm{E}} \mathrm{e}^{i \pi \cdot}\right\|_{\infty}=\pi$. Thus, we have $\left\|Q^{\mathrm{E}}\right\|=\pi$.

The next theorem shows that the mixed signal representation (8) converges uniformly on all of $\mathbb{R}$ to $Q^{\mathrm{E}} f$ for all $f \in \mathcal{B}_{\pi}^{\infty}$.

Theorem 2. For all $f \in \mathcal{B}_{\pi}^{\infty}$ we have

$$
\lim _{N \rightarrow \infty} \max _{t \in \mathbb{R}}\left|\left(Q^{E} f\right)(t)-\sum_{k=-N}^{N} a_{-k} f(t-k)\right|=0 .
$$

Proof. Let $f \in \mathcal{B}_{\pi}^{\infty}$. Then we have

$$
\begin{aligned}
& \left|\left(Q^{\mathrm{E}} f\right)(t)-\sum_{k=-N}^{N} a_{-k} f(t-k)\right|=\left|\sum_{|k|>N} a_{-k} f(t-k)\right| \\
& \leq \sum_{|k|>N}\left|a_{-k} f(t-k)\right| \leq\|f\|_{\infty} \sum_{|k|>N}\left|a_{-k}\right| .
\end{aligned}
$$

Since $\sum_{k=-\infty}^{\infty}\left|a_{k}\right|<\infty$, according to (11), it follows that $\lim _{N \rightarrow \infty} \sum_{|k|>N}\left|a_{-k}\right|=0$, and consequently we have (14) after taking the supremum on both sides of (15).

\section{THE HILBERT TRANSFORM FOR BOUNDED SIGNALS}

Despite the convergence problems discussed in Section 3 there is a way to define the Hilbert transform for signals in $\mathcal{B}_{\pi}^{\infty}$. This definition uses Fefferman's duality theorem, which states that the dual space of $\mathcal{H}^{1}$ is $\mathrm{BMO}(\mathbb{R})$ [14]. Before we give this definition, we briefly review some definitions.

Definition 1. The space $\mathcal{H}^{1}$ denotes the Hardy space of all signals $f \in L^{1}(\mathbb{R})$ for which $H f \in L^{1}(\mathbb{R})$. It is a Banach space endowed with the norm $\|f\|_{\mathcal{H}^{1}}:=\|f\|_{L^{1}(\mathbb{R})}+\|H f\|_{L^{1}(\mathbb{R})}$.

Definition 2. A function $f: \mathbb{R} \rightarrow \mathbb{C}$ is said to belong to $\operatorname{BMO}(\mathbb{R})$, provided that it is locally in $L^{1}(\mathbb{R})$ and $\frac{1}{\mu(I)} \int_{I}\left|f(t)-m_{I}(f)\right| \mathrm{d} t \leq$ $C_{2}$ for all bounded intervals $I$, where $m_{I}(f):=\frac{1}{\mu(I)} \int_{I} f(t) \mathrm{d} t$ and the constant $C_{2}$ is independent of $I . \mu$ denotes the Lebesgue measure.

For our further examinations, we need the important fact that the dual space of $\mathcal{H}^{1}$ is $\mathrm{BMO}(\mathbb{R})$ [15, p. 245]. In order to state this duality, we use the space $\mathcal{H}_{\mathrm{D}}^{1}=\mathcal{H}^{1} \cap \mathcal{S}$, which is dense in $\mathcal{H}^{1}$.

Theorem 3 (Fefferman). Suppose $f \in B M O(\mathbb{R})$. Then the linear functional $\mathcal{H}_{D}^{1} \rightarrow \mathbb{C}, \phi \mapsto \int_{-\infty}^{\infty} f(t) \phi(t) \mathrm{d} t$ has a bounded extension to $\mathcal{H}^{1}$. Conversely, every continuous linear functional $L$ on $\mathcal{H}^{1}$ is created as described before by a function $f \in B M O(\mathbb{R})$, which is unique up to an additive constant.

The function $f \in \operatorname{BMO}(\mathbb{R})$ in Theorem 3 is only unique up to an additive constant, because $\phi \in \mathcal{H}^{1}$ implies $\int_{-\infty}^{\infty} \phi(t) \mathrm{d} t=0$. Therefore, it will be beneficial to identify two functions in $\mathrm{BMO}(\mathbb{R})$ that differ only by a constant. We do this by introducing the equivalence relation $\sim$ on $\operatorname{BMO}(\mathbb{R})$. We write $f \sim g$ if and only if $f(t)=$ $g(t)+C_{\mathrm{BMO}}$ for almost all $t \in \mathbb{R}$, where $C_{\mathrm{BMO}}$ is a constant. By $[f]$ we denote the equivalence class $[f]=\{g \in \operatorname{BMO}(\mathbb{R}): g \sim f\}$, and $\operatorname{BMO}(\mathbb{R}) / \mathbb{C}$ is the set of all equivalence classes in $\operatorname{BMO}(\mathbb{R})$.

Next we extend the Hilbert transform, using the following definition, which is based on the $\mathcal{H}^{1}-\mathrm{BMO}(\mathbb{R})$ duality [9].

Definition 3. We define the Hilbert transform $\mathfrak{H} f$ of $f \in L^{\infty}(\mathbb{R})$ to be the function in $\operatorname{BMO}(\mathbb{R}) / \mathbb{C}$ that generates the linear continuous functional

$$
\langle\mathfrak{H} f, \phi\rangle=\int_{-\infty}^{\infty} f(t)(H \phi)(t) \mathrm{d} t, \quad \phi \in \mathcal{H}^{1} .
$$

Note that this definition is very abstract, because it gives no information how to calculate the Hilbert transform $\mathfrak{H} f$. In the next section we will provide an explicit formula for $\mathfrak{H} f$ that can be used to calculate the Hilbert transform. 


\section{EXTENSION OF THE HILBERT TRANSFORM}

In Section 4.2 we have seen that $Q^{\mathrm{E}}: \mathcal{B}_{\pi}^{\infty} \rightarrow \mathcal{B}_{\pi}^{\infty}$ is a bounded linear operator. Hence, for every $f \in \mathcal{B}_{\pi}^{\infty}$, the operator $\Im$ given by

$$
(\mathfrak{I} f)(t)=\int_{0}^{t}\left(Q^{\mathrm{E}} f\right)(\tau) \mathrm{d} \tau, \quad t \in \mathbb{R},
$$

is well defined. Since the operator $Q: \mathcal{P} \mathcal{W}_{\pi}^{2} \rightarrow \mathcal{P} \mathcal{W}_{\pi}^{2}$, as an operator on $\mathcal{P} \mathcal{W}_{\pi}^{2}$, was defined to be the concatenation of the Hilbert transform $H$ and the differential operator $D$, it is clear that, for $g \in$ $\mathcal{P} \mathcal{W}_{\pi}^{2}$, the integral of $Q g$ as in (16) gives-up to a constant- the Hilbert transform $\mathrm{Hg}$ of $g$. For $g \in \mathcal{P} \mathcal{W}_{\pi}^{2}$ we have

$$
\begin{aligned}
(\Im g)(t) & =\int_{0}^{t}\left(Q^{\mathrm{E}} g\right)(\tau) \mathrm{d} \tau=\int_{0}^{t}(Q g)(\tau) \mathrm{d} \tau \\
& =\int_{0}^{t}(D H g)(\tau) \mathrm{d} \tau=(H g)(t)-(H g)(0),
\end{aligned}
$$

i.e., for every signal $g \in \mathcal{P} \mathcal{W}_{\pi}^{2}$, we have $(H g)(t)=(\Im g)(t)+$ $C_{3}(g), t \in \mathbb{R}$, where $C_{3}(g)$ is a constant that depends on $g$.

Based on this observation one could conjecture that, for signals $f \in \mathcal{B}_{\pi}^{\infty}$, the integral $\mathfrak{I} f$ is somehow connected to the Hilbert transform $\mathfrak{H} f$ of $f$. The next theorem shows that such a connection exists in the sense that $\mathfrak{I} f$ is a representative of the equivalence class $\mathfrak{H} f$. This is an important result, because with $\mathfrak{I} f$ as defined in (16) we have an explicit formula to calculate the Hilbert transform of signals in $\mathcal{B}_{\pi}^{\infty}$. This is in contrast to Definition 3, which uses the abstract $\mathcal{H}^{1}-\mathrm{BMO}(\mathbb{R})$ duality.

Theorem 4. Let $f \in \mathcal{B}_{\pi}^{\infty}$. Then we have $\mathfrak{H} f=[\mathfrak{I} f]$.

The proof of Theorem 4 is omitted due to space constraints and will be published in an extended version of the paper [13].

An interesting consequence of Theorem 4 is Corollary 1.

Corollary 1. There exists a signal $g \in B M O(\mathbb{R})$ such that $\mathfrak{H} f=[g]$ and $|g(t)| \leq \pi\|f\|_{\infty}|t|$ for all $t \in \mathbb{R}$.

This corollary gives an upper bound on the growth of the Hilbert transform $\mathfrak{H} f$. It shows that $\mathfrak{H} f$ is locally bounded and that the growth on the real axis is at most linear. We do not know whether this upper bound is the best possible.

The next corollary shows that $\mathfrak{I} f$ is a bandlimited signal for every $f \in \mathcal{B}_{\pi}^{\infty}$. It immediately follows that the Hilbert transform $\mathfrak{H} f$ of a bounded bandlimited signal $f \in \mathcal{B}_{\pi}^{\infty}$ is bandlimited in a sense which is stated more precisely in Corollary 3 .

Corollary 2. Let $f \in \mathcal{B}_{\pi}^{\infty}$. Then we have $\mathfrak{I} f \in \mathcal{B}_{\pi}$.

Proof. Let $f \in \mathcal{B}_{\pi}^{\infty}$. Then we have the mixed signal representation (13) of $Q^{\mathrm{E}} f$. Since, according to Theorem 2, the series is uniformly convergent, it follows that $\left(Q^{\mathrm{E}} f\right)(t)$ can be extended to an entire function $\left(Q^{\mathrm{E}} f\right)(z), z \in C$. Further, we have

$$
\left|\left(Q^{\mathrm{E}} f\right)(z)\right| \leq\|f\|_{\infty} \mathrm{e}^{\pi|\operatorname{Im}(z)|} \sum_{k=-\infty}^{\infty}\left|a_{-k}\right|=\pi\|f\|_{\infty} \mathrm{e}^{\pi|\operatorname{Im}(z)|}
$$

because $|f(z-k)| \leq\|f\|_{\infty} \mathrm{e}^{\pi|\operatorname{Im}(z)|}$ for all $z \in \mathbb{C}[16]$, and because of (11). Hence, it follows that

$$
|(\Im f)(z)|=\left|\int_{0}^{z}\left(Q^{\mathrm{E}} f\right)(\xi) \mathrm{d} \xi\right| \leq|z| \pi\|f\|_{\infty} \mathrm{e}^{\pi|\operatorname{Im}(z)|} .
$$

Thus, for every $\epsilon>0$ there exists a constant $C_{4}=C_{4}(\epsilon)$ such that $|(\Im f)(z)| \leq C_{4} \mathrm{e}^{(\pi+\epsilon)|z|}$ for all $z \in \mathbb{C}$.
Corollary 3. Let $f \in \mathcal{B}_{\pi}^{\infty}$. Then there exists a signal $g \in$ $B M O(\mathbb{R}) \cap \mathcal{B}_{\pi}$ such that $\mathfrak{H} f=[g]$.

Corollaries 1 and 3 imply that the Hilbert transforms $\mathfrak{H} f$ of signals $f \in \mathcal{B}_{\pi}^{\infty}$ is in the Zakai class. Thus, with the proposed extension of the Hilbert transform, concepts like the analytical signal can still be used for signals in $\mathcal{B}_{\pi}^{\infty}$.

Since signals $f \in \mathrm{BMO}(\mathbb{R})$ are not necessarily bounded, it cannot be expected that the extended Hilbert transform $\mathfrak{H} f$ of a signal in $L^{\infty}(\mathbb{R})$ is again in $L^{\infty}(\mathbb{R})$. However, if the signal $f$ is additionally bandlimited the situation might be different. The next theorem shows that this is not the case.

Theorem 5. There exists a signal $f_{1} \in \mathcal{B}_{\pi}^{\infty}$ such that every representative of he equivalence class $\mathfrak{H} f_{1}$ is unbounded on the real axis.

In Corollary 3 we have seen that the Hilbert transform of a bounded bandlimited signal in $\mathcal{B}_{\pi}^{\infty}$ is still bandlimited in a certain sense. However, as Theorem 5 shows, the boundedness property of a $\mathcal{B}_{\pi}^{\infty}$ signal is not preserved under the Hilbert transform.

\section{REFERENCES}

[1] D. Gabor, "Theory of communication," Journal of the Institute of Electrical Engineers, vol. 93, no. 3, pp. 429-457, Nov. 1946.

[2] H. B. Voelcker, "Toward a unified theory of modulation part I: Phase-envelope relationships," Proceedings of the IEEE, vol. 54, no. 3, pp. 340-353, March 1966.

[3] L. M. Fink, "Relations between the spectrum and instantaneous frequency of a signal," Problems of Information Transmission, vol. 2, no. 4, pp. 11-21, 1966, translation.

[4] D. Ye. Vakman, "On the definition of concepts of amplitude, phase and instantaneous frequency of a signal," Radio Eng. Electron. Phys., vol. 17, no. 5, pp. 754-759, 1972, translation.

[5] V. Pohl and H. Boche, Advanced Topics in System and Signal Theory: A Mathematical Approach, Springer, 2009.

[6] R. P. Boas, Jr., "Some theorems on Fourier transforms and conjugate trigonometric integrals," Transactions of the American Mathematical Society, vol. 40, no. 2, pp. 287-308, 1936.

[7] B. F. Logan, Jr., "Theory of analytic modulation systems," Bell Syst. Tech. J., vol. 57, no. 3, pp. 491-576, March 1978.

[8] F. W. King, Hilbert Transforms: Volume 2, Cambridge University Press, 2009.

[9] Lihua Yang and Haizhang Zhang, "The Bedrosian identity for $H^{p}$ functions," Journal of Mathematical Analysis and Applications, vol. 345, no. 2, pp. 975-984, September 2008.

[10] V. I. Korzhik, "The extended Hilbert transformation and its application in signal theory," Problems of Information Transmission, vol. 5, no. 4, pp. 1-14, 1969, translation.

[11] J. L. Brown, Jr., "Analytic signals and product theorems for Hilbert transforms," IEEE Transactions on Circuits and Systems, vol. 21, no. 6, pp. 790-792, November 1974.

[12] L. Grafakos, Classical Fourier Analysis, Springer, 2 ed., 2008.

[13] H. Boche and U. J. Mönich, "On the Hilbert Transform of Bounded Bandlimited Signals," to be published, 2012.

[14] C. Fefferman, "Characterization of bounded mean oscillation," Bull. Amer. Math. Soc., vol. 77, no. 4, pp. 587-588, July 1971.

[15] J. B. Garnett, Bounded Analytic Functions, Academic Press, 1981.

[16] Richard Duffin and A. C. Schaeffer, "Some properties of functions of exponential type," Bull. Amer. Math. Soc., vol. 44, no. 4, pp. 236-240, April 1938. 